\title{
La importancia de la sesión grupal en la clase inversa: Trabajos colaborativos en una asignatura de Matemáticas de Grado durante el curso 2016-2017
}

\author{
Anna Vidal Meló, Francisco J. Boigues Planes, Vicente D. Estruch Fuster ${ }^{(3)}$ \\ Grup Grup d'Innovació Educativa i Recerca en Màteries Cientifiques \\ Departamento de Matemática Aplicada, Universitat Politècnica de València, Escuela \\ Politécnica Superior de Gandia, C/ Paranimf, 1, 46730 Grau de Gandia (Valencia) \\ avidal@mat.upv.es fraboipl@mat.upv.es vdestruc@mat.upv.es
}

Presentado en eXIDO17 (2017)

\begin{abstract}
RESUMEN
En este trabajo se describen algunos de los trabajos realizados durante el curso 20162017 por los estudiantes del Grado en Ingeniería de Sistemas de Telecomunicación, Sonido e Imagen de la Universitat Politècnica de València, en las prácticas de una asignatura anual del primer año, Matemáticas 2. La utilización de la Clase Inversa en dichas prácticas ha permitido dedicar las horas presenciales a la realización de trabajos colaborativos durante la sesión presencial o grupal. Se ha utilizado la plataforma PoliformaT a través de la cual profesores y estudiantes pueden compartir información sobre sus asignaturas así como utilizar herramientas para su gestión como son tareas, exámenes o la herramienta Lessons que permite la creación de contenidos e itinerarios formativos, facilitándonos la implementación de la clase inversa en esta asignatura. Durante las prácticas nuestros estudiantes han utilizado el programa Matlab consiguiendo entre todos varios productos finales, como una colección de gráficas de curvas en coordenadas paramétricas y polares, una colección de gráficas de superficies y una representación tridimensional de la Sierra de Bernia. Durante el curso se han realizado varios posters de estos resultados conjuntos que han sido expuestos en nuestro aulario, despertando el interés de profesores y estudiantes de otras asignaturas.
\end{abstract}

Palabras clave: trabajo colaborativo, clase inversa, Matemáticas

\section{INTRODUCCIÓN}

Matemáticas 2 es una asignatura anual del primer curso del Grado en Ingeniería de Sistemas de Telecomunicación, Sonido e Imagen (GISTSI), impartida en el Campus de Gandia de la Universitat Politècnica de València (UPV). La asignatura tiene asignados 9 ECTS, distribuidos en los siguientes bloques:

Bloque I: Integración en una variable

Tema 1: Integración de funciones de una variable

Tema 2: Aplicaciones de las integrales

Bloque II: Series

Tema 3: Series numéricas

Tema 4: Series de potencias y representación en serie de potencias

Bloque III: Cálculo diferencial en funciones de varias variables

Tema 5: Estudio de las funciones de varias variables

Tema 6: Introducción a la optimización 
Bloque IV: Integración de funciones de varias variables

Tema 7: Integral doble y triple

Bloque V: Fundamentos matemáticos con Matlab

Matlab y matemáticas

Curvas en paramétricas y en polares

Interpolación y ajuste

Integración numérica

Representación de campos escalares: formas cartesiana y paramétrica

Representación de superficies en forma discreta

El último bloque se corresponde con las prácticas (informáticas) que se realizan en sesiones de dos horas cada una. Estas, junto con una práctica de evaluación, suponen 1.4 ECTS de la asignatura y un $20 \%$ de la nota de esta. El programa utilizado en dichas prácticas es Matlab, al que nos tuvimos que adaptar el profesorado de Matemáticas por ser el programa utilizado en la mayoría de las asignaturas de la titulación.

En general, y aunque estas prácticas están muy relacionadas con el temario de teoría, su contenido teórico sólo se estudia en las sesiones prácticas. Así, por ejemplo, el estudio de otras curvas que no sean las que se deducen de gráficas de funciones de una variable, es decir, el estudio de las curvas paramétricas o polares, solo se estudia en la sesión práctica, previo al tema 2 sobre las aplicaciones de las integrales, pudiendo calcular áreas, volúmenes de sólidos de revolución o longitudes también de estas nuevas curvas en dicho tema. La teoría y aplicaciones de la práctica de integración numérica completa también el estudio de este segundo tema. En cuanto a las dos prácticas sobre representación de campos escalares complementan los temas 5, 6 y 7.

En años anteriores se dedicaban las dos horas presenciales de prácticas al estudio de la teoría asociada a cada práctica, junto con alguna resolución sencilla y guiada de algún problema. El alumnado debía repetir lo que hacía el profesor en clase siguiendo una guía detallada en formato .pdf. Posteriormente se les mandaba un trabajo en grupo que debían resolver fuera de las clases presenciales, en un plazo máximo de 10 días. A lo largo de los años se observaron una serie de problemas en la realización de estos trabajos y entre los miembros del grupo, lo que condujo a que nos planteáramos un cambio de metodología.

El cambio nos lo proporcionó la Clase Inversa o Flipped Classroom. Desde el curso 2014-2015 esta metodología se ha inmerso en un proyecto institucional de nuestra universidad, que pretende "avanzar en la implantación de innovaciones metodológicas en los procesos formativos, enfatizando el desarrollo de métodos de aprendizaje para la adquisición de competencias por parte del estudiante y procedimientos e instrumentos para su evaluación” (http://claseinversa.blogs.upv.es/el-proyecto/proyecto-claseinversa-upv-2/).

La Clase Inversa tiene sus orígenes en el método del caso utilizado en las escuelas de negocios, derecho y humanidades, con el que se exigía al alumnado que, previo a la clase, prepararan una lectura para establecer en clase una discusión (Lage, Platt y Treglia, 2000). Más tarde, en 2007, dos profesores de química en Woodland Park High School, Colorado, Jonathan Bergmann y Aaron Sams, empezaron a grabar los contenidos de sus asignaturas en video y a subirlos a la red, siendo los primeros en defender esta herramienta como instrumento de enseñanza (Bergamnn y Sams, 2105). 
La Clase Inversa está basada en la idea central de "dar la vuelta” o alterar el orden de las actividades de la enseñanza tradicional. De forma muy básica, el aprendizaje empieza fuera del aula con la utilización del material seleccionado por el docente, dedicando posteriormente la sesión presencial o grupal a la resolución de ejercicios, proyectos y discusiones.

En la Figura 1 se observa el cambio de rol entre el profesorado y el alumnado en una clase inversa relacionando cada una de las actividades con la taxonomía revisada de Bloom.

\section{Clase tradicional}

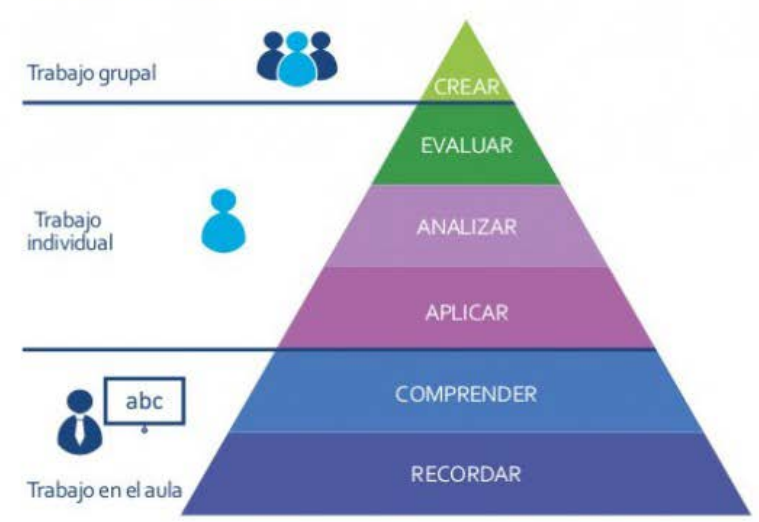

Flipped classroom

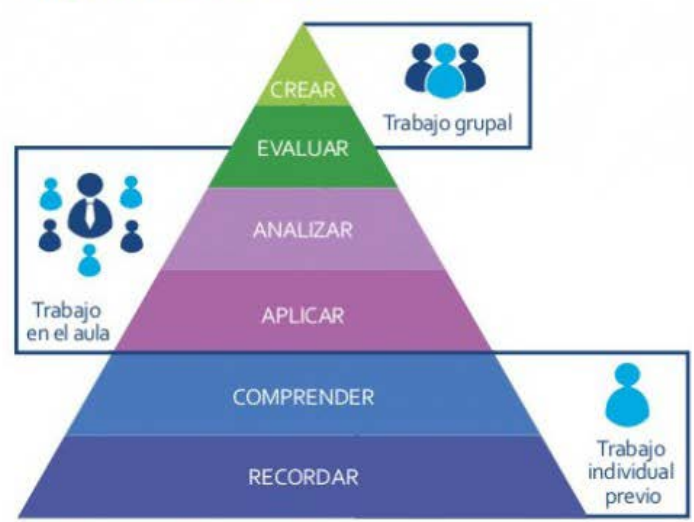

Figura 1. Infografía relacionando la clase tradicional y la clase inversa, apoyadas con la taxonomía revisada de Bloom (Fuente: aulaplaneta.com)

Esta técnica busca despertar actitudes que involucran al alumno de forma positiva en la asignatura, fundamentalmente el aprendizaje activo y el compromiso personal, aprovechando mucho más el tiempo en la clase presencial que se convierte en un "taller de experiencias", donde los estudiantes puedan recibir feedback por parte del profesor o de los compañeros, evaluar sus propias habilidades, aplicar los conocimientos y habilidades adquiridos previamente e interactuar mediante actividades en grupo. Exige por parte del estudiante dedicación y compromiso y por parte del profesor la selección y elaboración de buenos materiales, el diseño de actividades de aprendizaje significativas, tanto para la sesión no presencial como para la sesión grupal, siendo de suma importancia el trabajo en el aula. Así pues, uno de los elementos más importantes y complejos para que esta técnica sea un éxito lo constituye el establecer un diseño adecuado de la sesión presencial, pudiendo incluir por ejemplo resolución de problemas o actividades de simulación bien en grupo o de manera individual, pero en cualquier caso con la característica común de que el profesor actúa como guía del aprendizaje es decir como facilitador para que los estudiantes asuman la responsabilidad activa en su experiencia de aprendizaje y que adquieran las competencias establecidas con la capacidad de poderlas poner en práctica en otros contextos.

Las competencias relacionadas con el trabajo en el aula, es decir, de la sesión presencial o grupal, son de suma importancia para el aprendizaje del alumnado: aplicar, analizar y evaluar. En esta sesión se conoce si el estudiante está aprendiendo y va a ser 
fundamental tanto la metodología utilizada en dicha sesión como el diseño de las actividades a realizar, como puede ser un trabajo en grupo.

En este trabajo se muestra el modelo seguido en las sesiones presenciales de las prácticas de Matemáticas 2 y detallamos los trabajos colaborativos planteados y desarrollados por nuestro alumnado, trabajos planteados como un puzle, en el sentido de que el trabajo de cada uno de los grupos es significativo para la obtención de un producto final, como veremos posteriormente.

En todo este proceso también ha sido fundamental el disponer de la plataforma de teleformación, o aula virtual de la UPV, utilizada para desarrollar nuestra docencia, PoliformaT. A través de esta profesores y estudiantes comparten información acerca de sus asignaturas a través de varias herramientas de gestión de las que destacamos las siguientes:

- Exámenes: permite crear y generar exámenes tipo test para que el estudiante pueda hacerlos tanto en la sesión presencial como en la no presencial, en un tiempo limitado si se desea.

- Tareas: utilizada en nuestro caso para los trabajos en grupo de forma que el alumnado tenga a su disposición en este apartado, tanto la documentación informativa sobre el trabajo a realizar como la memoria a entregar dentro de un plazo concretado.

- Lessons: permite crear contenido digital interactivo, generar unidades didácticas, insertar vídeos, programar actividades y enlazar con Tareas y Exámenes. En la Figura 2 puede verse parte del Lessons correspondiente a la práctica de integración numérica.

En la siguiente sección analizamos de forma detallada la metodología seguida durante los cursos 2015-16 y 2016-17 en las prácticas informáticas a las que se refiere este trabajo. 


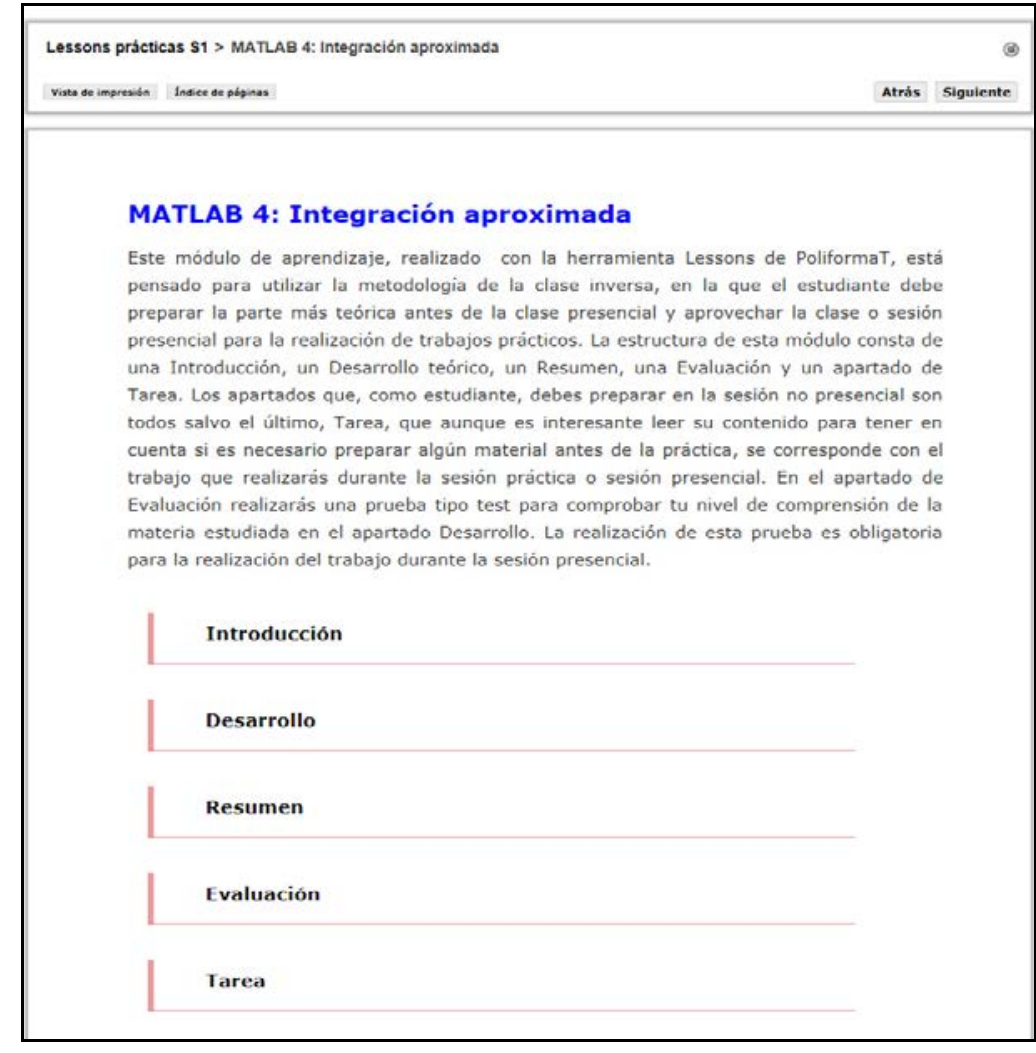

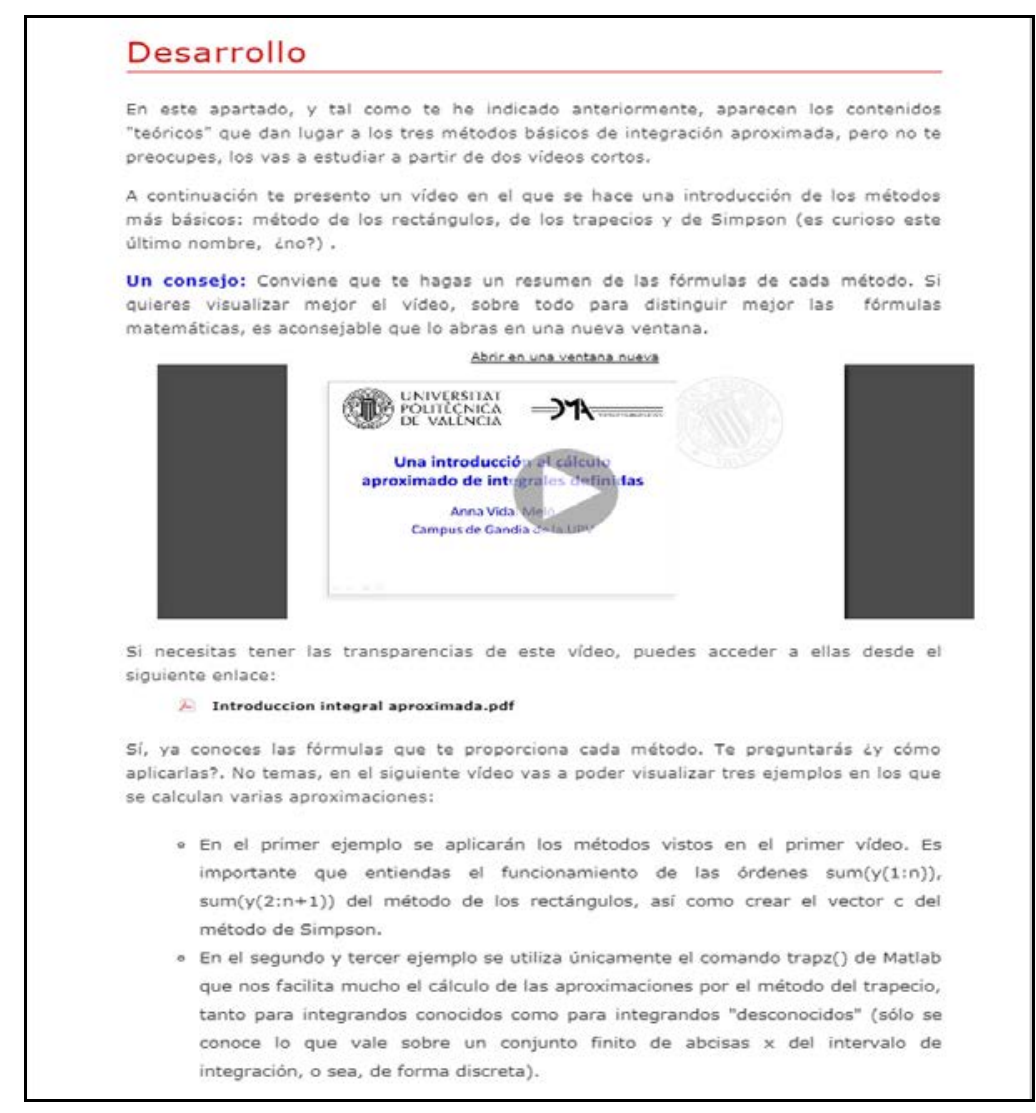

Figura 2. Modelo de Lessons para la práctica de integración aproximada 


\section{METODOLOGÍA}

En la sección anterior se ha descrito el proceso seguido hasta el curso 2014-15 en las prácticas de la asignatura Matemáticas 2. Con la adopción de la Clase Inversa, se ha tenido que generar material nuevo, en concreto un Lessons para cada una de las prácticas y en particular para las prácticas asociadas a los trabajos colaborativos que describiremos en este trabajo:

Curvas en paramétricas y en polares

Integración numérica

Representación de campos escalares: formas cartesiana y paramétrica

Representación de superficies en forma discreta

En algunos casos también se han realizado vídeos de corta duración (Polimedias) o screencast. Así, y como ejemplo, en la práctica sobre integración aproximada se han realizado dos Polimedias. En el primero se explican los métodos básicos de integración aproximada (rectángulos, trapecios y Simpson) junto con cada una de sus fórmulas que van a permitir ser programadas en Matlab para aplicarlas a casos y ejemplos concretos en el segundo vídeo.

Los Lessons generados constituyen una unidad en cuanto a que contienen tanto la parte del desarrollo teórico como un enlace a Exámenes a preparar en la sesión no presencial o individual junto con un enlace a la herramienta Tareas correspondiente a la sesión presencial o grupal.

En cuanto a la metodología seguida en cada una de las sesiones prácticas, distinguiremos entre las actividades correspondientes al trabajo no presencial o sesión individual de la sesión práctica o grupal.

\section{Trabajo no presencial previo (sesión individual):}

Es la correspondiente a las actividades que debe realizar el alumnado en casa, previa a la sesión de prácticas. El estudiante prepara el contenido "teórico" de la práctica con el correspondiente Lessons, y realiza un examen test sobre lo estudiado. La realización de este examen es obligatoria para poder realizar con posterioridad con éxito la práctica y el trabajo correspondiente y debe hacerse con un tiempo limitado, hasta la tarde antes del día de la práctica. Esta limitación en tiempo nos permite primero controlar qué estudiantes han cumplido con la tarea no presencial y en segundo lugar conocer si han entendido bien o no los apartados teóricos.

\section{Clase presencial, sesión práctica (sesión grupal):}

Esta sesión tiene una duración de 2 horas. Durante los primeros 15 minutos el profesorado muestra y explica los resultados del test, haciendo hincapié en aquellas preguntas con peores resultados y resolviendo las posibles dudas. Esta parte es de suma importancia ya que de esta forma se le proporciona al alumnado el feedback necesario. A continuación, y a través del enlace a Tareas insertado en el Lessons, se muestran los documentos correspondientes al trabajo que por grupos de 2 o 3 alumnos deben realizar durante el resto de la sesión práctica y cuya memoria dejarán en la correspondiente Tarea al finalizar dicha sesión práctica. 


\section{LOS TRABAJOS}

En esta sección describimos cada uno de los trabajos colaborativos correspondientes a las prácticas citadas en el apartado de Metodología y sus resultados.

\section{Curvas en paramétricas y en polares}

El objetivo final de esta práctica es obtener catálogo formado por un buen número de representaciones de curvas en ambas coordenadas. Anteriormente a la adopción de la Clase inversa también se realizaba este trabajo en grupo (Vidal, 2013), aunque fuera de la sesión presencial. Previo a la realización de la práctica, y además del trabajo autónomo correspondiente al estudio del Lessons, a cada grupo de estudiantes se le asigna una yincana en la que deben encontrar tres curvas escondidas. Las dos primeras se encuentran en algunos libros de nuestra biblioteca y en la yincana aparecen pistas del título de los libros, páginas y ejercicios o ejemplos en donde localizarlas. La tercera se puede encontrar en alguna página de internet y para ello se le dan las pistas correspondientes. Los estudiantes deben anotar las ecuaciones de las curvas escondidas y traerlas a la sesión grupal en la que las representaban utilizando una plantilla común, obteniendo representaciones como las de la Figura 3. Hay que señalar que se tienen aproximadamente 30 yincanas distintas que hacen que cada grupo trabaje con curvas distintas. Este curso se les ha pedido además a los estudiantes un selfi que demostrara su descubrimiento en la biblioteca (Figura 4). El catálogo durante este curso ha sido en forma de póster (Figura 5) y ha sido expuesto en el aula de clase.

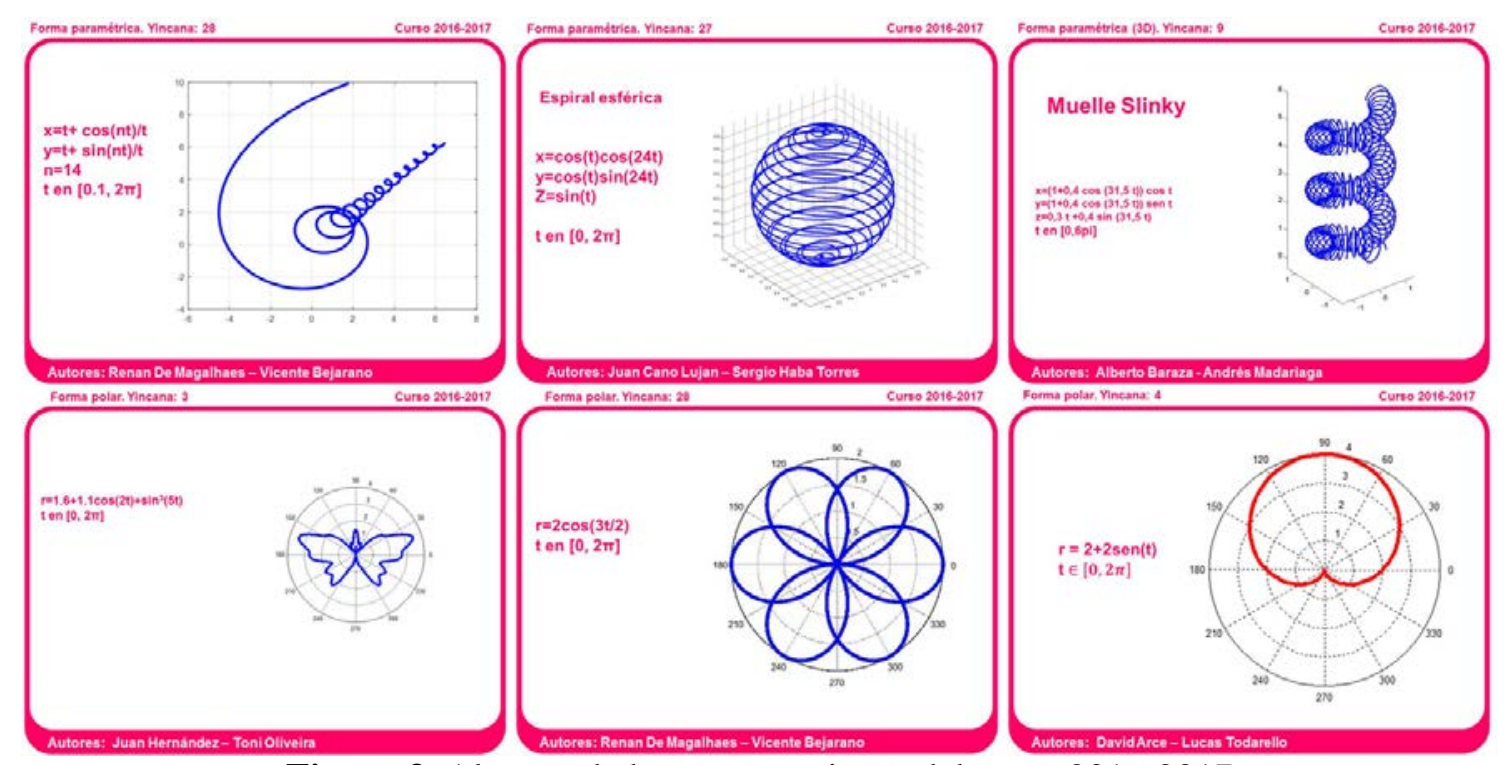

Figura 3. Algunas de las maquetaciones del curso 2016-2017
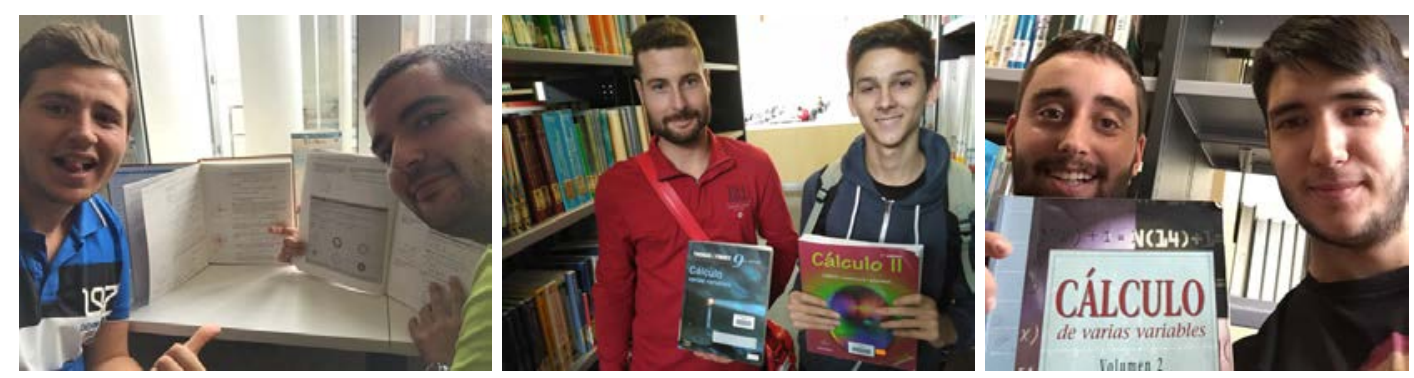
Figura 4. Algunos selfis de los estudiantes

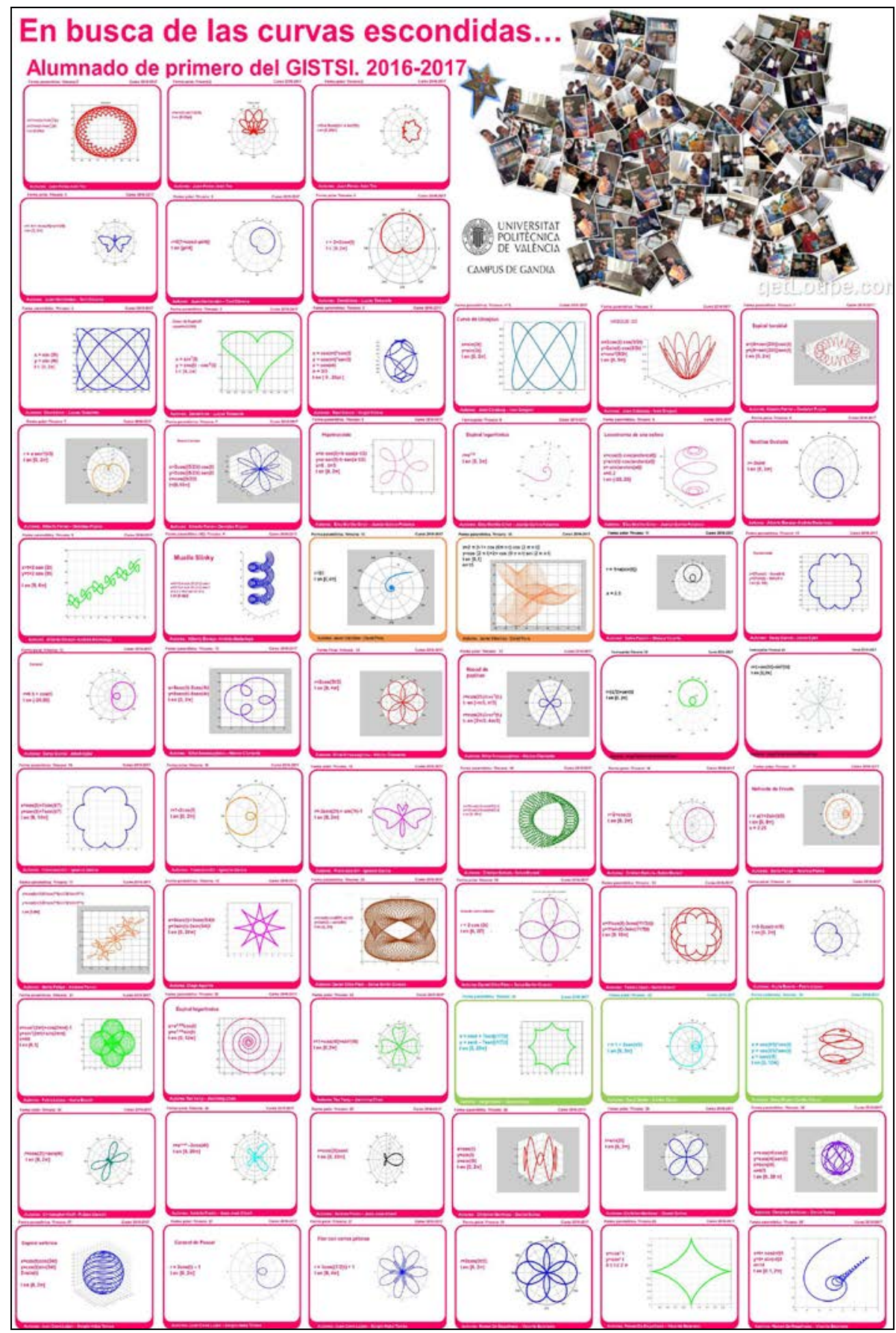

Figura 5. Catálogo en formato póster de las curvas paramétricas y polares

\section{Integración numérica}

El trabajo propuesto ha consistido en determinar una aproximación del volumen de la porción de un relieve, a través de la integral del área $A(x)$ de sus secciones transversales o curvas de nivel, integral dada por 


$$
V=\int_{a}^{b} A(x) d x
$$

El método requerido es el método de integración aproximada por trapecios de Matlab a través del comando trapz, comando que no requiere una partición equidistante. Para ello es necesario determinar las áreas de un conjunto finito de curvas de nivel. Google Maps nos permite obtener un mapa topográfico del relieve correspondiente y asignar a cada grupo de trabajo una curva de nivel concreta para que determinen una aproximación del área que encierra a través de varias mediciones de su anchura, como puede verse en la Figura 6.

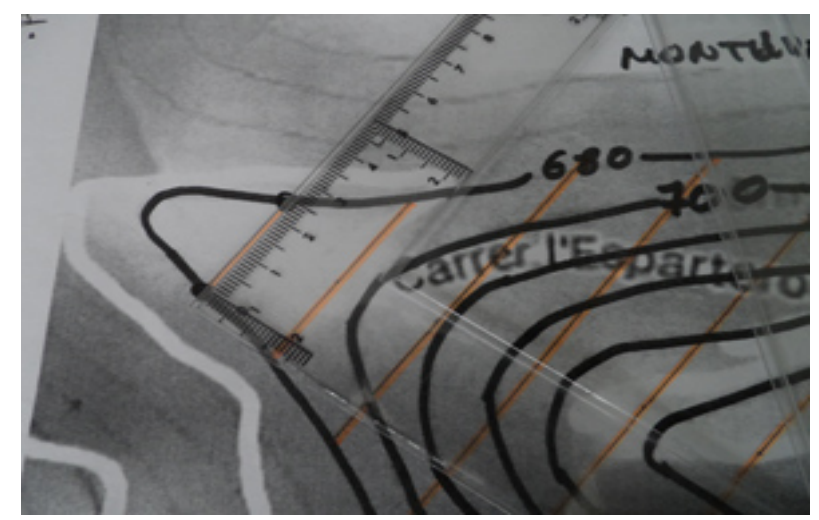

Figura 6. Midiendo anchuras de una curva de nivel

Con la ayuda del comando trapz de Matlab cada grupo obtiene un valor aproximado de $A(N)$, para alguno de los valores $N=x_{1}, x_{2}, \ldots, x_{n}$, siendo $a=x_{1}<x_{2}<\ldots<x_{n}=b$. El profesor recopila los resultados de cada grupo y, teniendo en cuenta la escala del mapa, se obtiene una aproximación del volumen aplicando de nuevo el método de los trapecios para la integral (1).

Durante el curso 2016-2017 se ha realizado el estudio del Monte Mayón (Filipinas) entre 1000 m y 2400 m, y del Puig Campana (Alicante) entre 900 m y 1360 m. Para el primero de ellos el cálculo del volumen aproximado ha resultado ser de $2124200000 \mathrm{~m}^{3}$ y en el segundo caso de $108550000 \mathrm{~m}^{3}$.

\section{Representación de campos escalares: formas cartesiana y paramétrica}

El objetivo es similar al de la práctica de curvas paramétricas y polares, es decir, obtener un catálogo de superficies. A cada grupo se le asigna un listado de 4 superficies a representar. La primera se corresponde con las diversas representaciones de la gráfica de una función $f(x, y)$ a través de su gráfica, de sus secciones transversales o líneas de contorno, el mapa de curvas de nivel y finalmente el mapa de color. La segunda figura se corresponde con la representación de una superficie paramétrica. La tercera es una superficie de revolución que deben generar a partir de una curva generatriz y su eje de giro, representando tanto la superficie cerrada como la abierta (limitando el ángulo de giro). Finalmente la cuarta figura se corresponde con la generación de una superficie 
cilíndrica a partir de las curvas obtenidas en la práctica de curvas paramétricas y polares. En la Figura 7 se muestran algunos resultados.
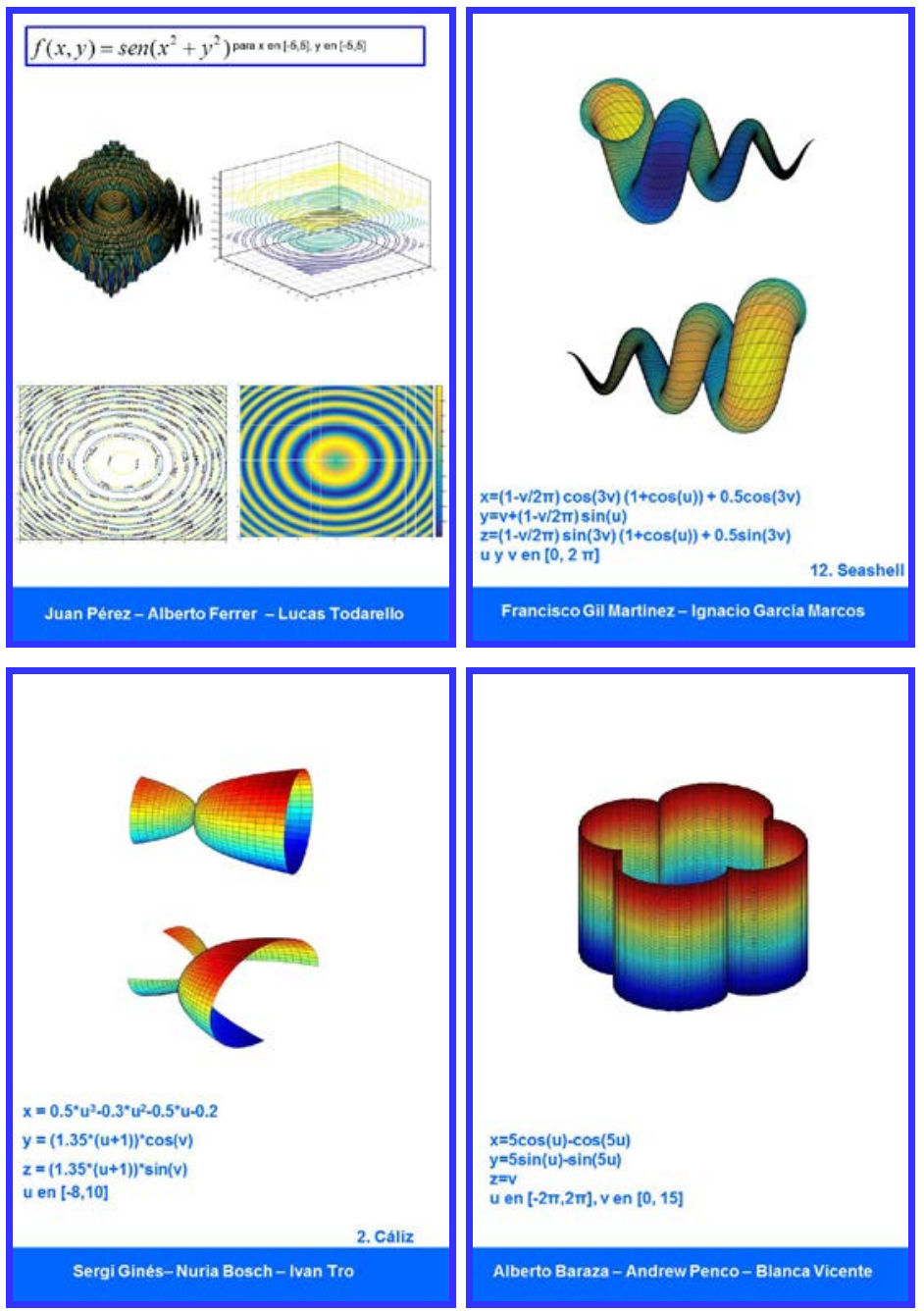

Figura 7. Alguna de las superficies representadas

Por último lanzamos el concurso "Disfraza una superficie" en el que además de representar una superficie, le debían añadir textura, siendo las ganadoras las representadas en la Figura 8. De nuevo en este caso se realizó el catálogo final en formato póster (Figura 9) que fue expuesto en el aulario de nuestro centro junto con las figuras participantes en el citado concurso, tal y como se aprecia en la Figura 10, hecho que resultó de interés tanto para profesores como para alumnos del GISTSI y de otras titulaciones. 

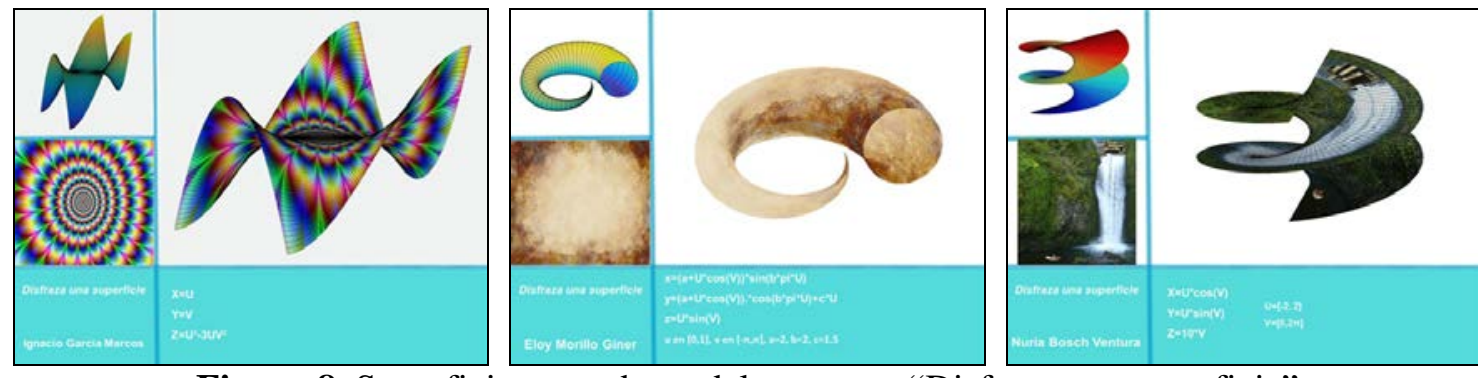

Figura 8. Superficies ganadoras del concurso "Disfraza una superficie"

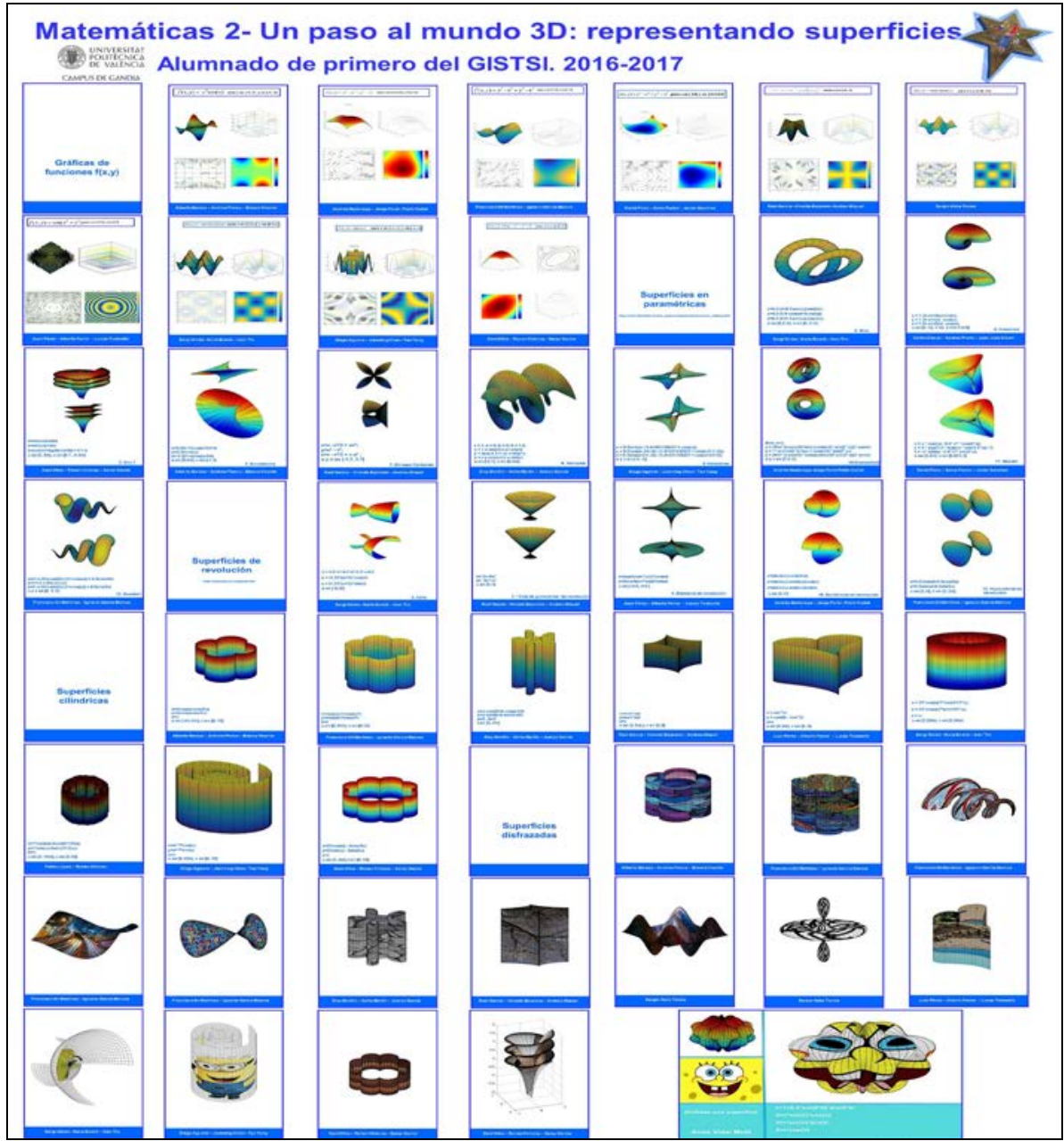

Figura 9. Catálogo en formato póster de las superficies

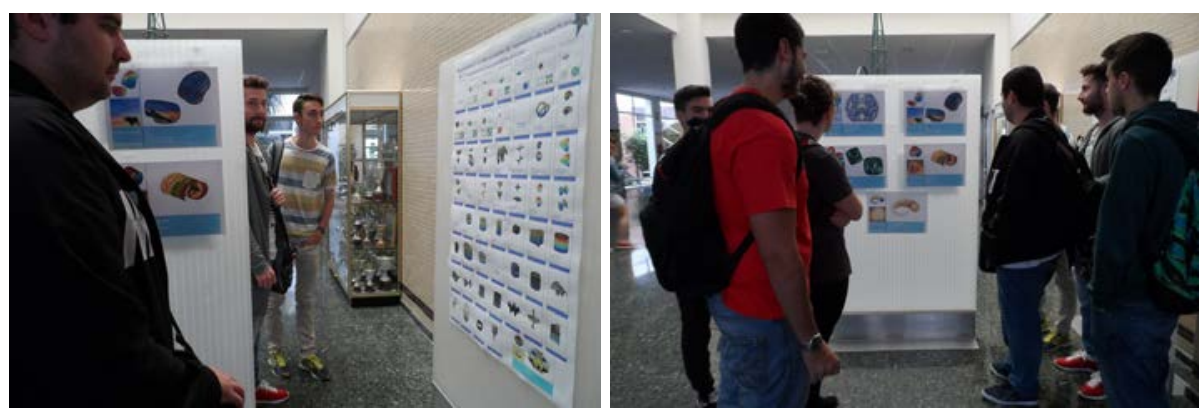

Figura 10. Exposición del póster junto con las figuras del concurso 


\section{Representación de superficies en forma discreta}

Cuando se desconocen las ecuaciones de los puntos $(x, y, z)$ de una superficie pero son conocidos un conjunto finito de sus puntos $\left(x_{i}, y_{i}, z_{i}\right), i=1,2, \ldots n$, Matlab permite obtener una representación de la superficie interpolada. En el caso particular de puntos $\left(x_{i}, y_{i}\right), i=1,2, \ldots n$ correspondientes a los nodos de una malla rectangular, se puede representar un primer boceto de la superficie y posteriormente, a través de la interpolación que realiza Matlab con el comando interp2, obtener la interpolada mejorada.

En nuestro caso la superficie a estudiar y representar en este trabajo colaborativo fue la Sierra de Bernia, de la provincia de Alicante. Esta sierra es elegida por muchos senderistas por su forat o agujero (Figura 11 ) que suele ser atravesado a gatas desde su cara norte para ir abriéndose en una especie de cueva con hiedras y encontrar en frente el mar, la Sierra Helada, Altea y Benidorm.

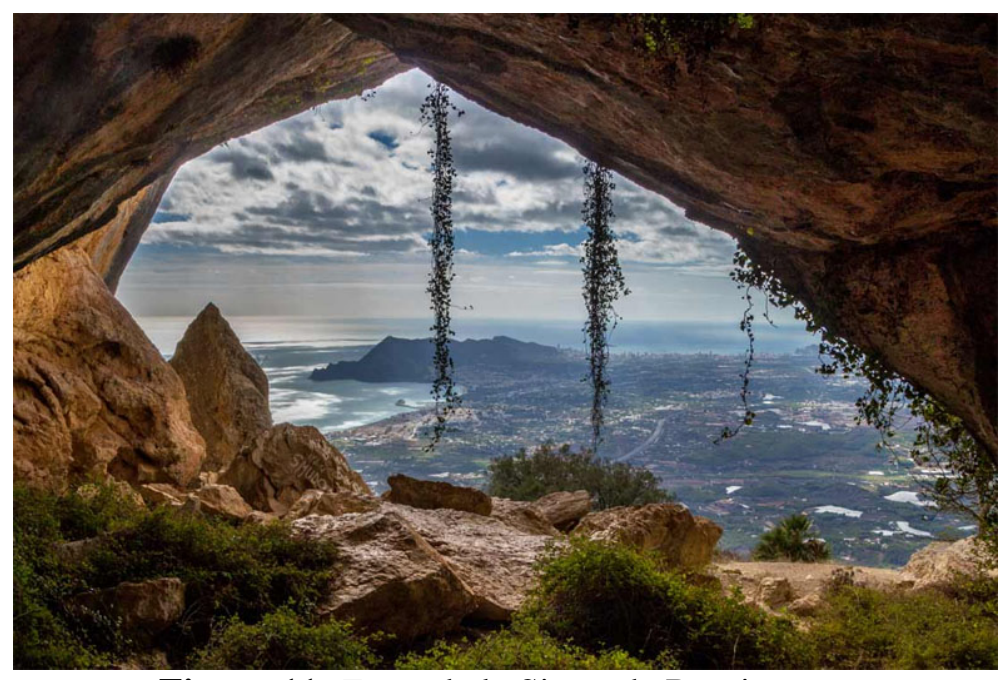

Figura 11. Forat de la Sierra de Bernia

(Fuente: https://tripkay.com/destination-guides/wp-content/uploads/2016/03/sierra-de-berniaen-altea.jpg )

De nuevo se ha recurrido a Google Maps para obtener un mapa topográfico y dividirlo en secciones para que cada uno de los grupos de trabajo estudie una de ellas. En la Figura 12 puede observarse la división establecida y la asignación a cada uno de los grupos. Cada grupo discretiza su sección tomando las medidas de las alturas $z_{i j}$ sobre los nodos $\left(x_{i}, y_{j}\right)$ de la malla rectangular establecida, malla similar a la de la Figura 13. Una vez definida la matriz de las alturas medidas cada uno de los grupos obtiene un primer boceto de la representación en 3D de su sección y con el comando interp2 la superficie interpolada. A partir del trabajo de cada uno de los grupos el profesorado genera la superficie total, tanto la medida inicialmente como la interpolada. El resultado final de este trabajo colaborativo ha sido de nuevo la creación de un póster, como se aprecia en la Figura 14 que incluía tanto la representación por secciones como la global. 


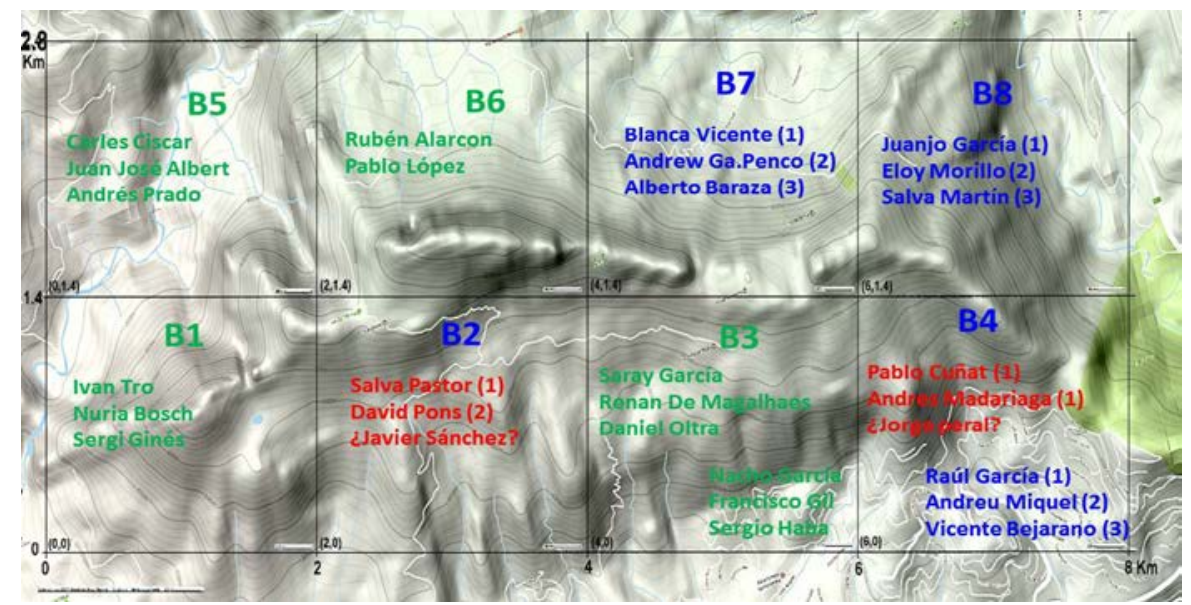

Figura 12. Mapa topográfico de la Sierra de Bernia y divisiones establecidas

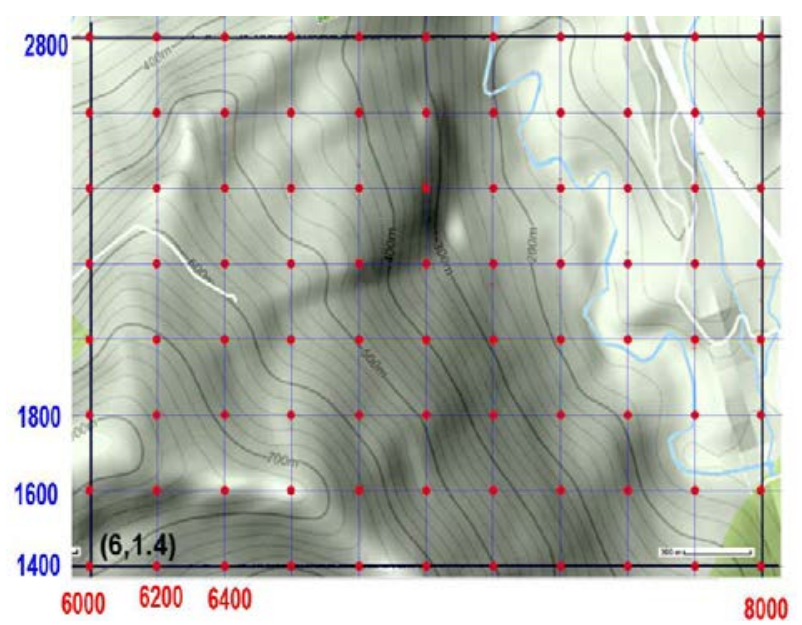

Figura 13. Malla rectangular y nodos establecidos en una de las secciones a estudiar

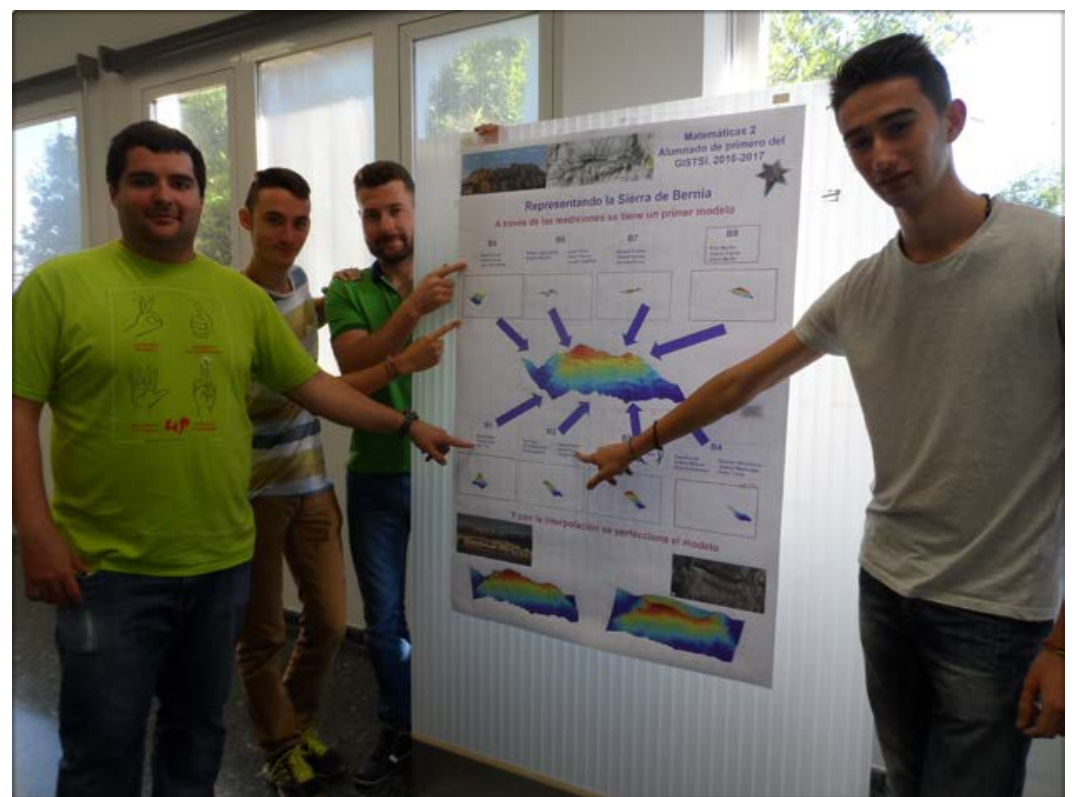

Figura 14. Póster final de la Sierra de Bernia y algunos de los estudiantes indicando su sección de estudio 


\section{CONCLUSIONES}

Nuestra experiencia en la aplicación de la Clase Inversa nos indica la importancia que tiene la buena selección de materiales y actividades a realizar tanto en la sesión no presencial o individual como en la presencial o grupal. En particular una buena planificación de la sesión de las prácticas de nuestra asignatura Matemáticas 2, ha permitido la obtención de los resultados descritos.

La Clase Inversa facilita la adquisición de algunas competencias transversales y su evaluación: Planificación y gestión del tiempo, liderazgo y trabajo en equipo, aprendizaje autónomo. Por otra parte esta metodología ha permitido la realización de los trabajos colaborativos en la sesión presencial de las prácticas informáticas de la asignatura, de forma que los estudiantes tienen la ayuda del profesor, evitándose además problemas de coordinación entre los miembros del grupo. Los trabajos prácticos descritos han resultado motivadores para nuestros estudiantes, y más el hecho de haber expuesto el resultado de estos en la clase o en el aulario. Este hecho también ha permitido dar a conocer a otros profesores y alumnado la labor que están haciendo nuestros estudiantes en la asignatura.

\section{AGRADECIMIENTOS}

Este trabajo ha sido financiado por la ayuda del Vicerrectorado de Estudios, Calidad y Acreditación de la Universitat Politècnica de València (PIME 2016-2017 "Puesta en marcha de diversas experiencias con el enfoque Flipped Teaching en asignaturas de Matemáticas y Física”) y por la ayuda a Proyectos de Innovación Docente del Dpto. de Matemática Aplicada de la UPV (PID-DMA-2017 "El papel de la clase presencial en la metodología Flip”).

\section{REFERENCIAS}

aulaPlaneta (2017). Ideas y recursos para poner tu clase al revés con la 'flipped classroom'. Recuperado de: http://www.aulaplaneta.com/2014/07/29/recursos-tic/ideas-y-recursospara-poner-tu-clase-del-reves-con-la-flipped-classroom/

Bergmann, J. Y Sams, A. (2015). Dale la vuelta a tu clase: Lleva tu clase a cada estudiante, en cualquier momento y cualquier lugar. SM.

Clase Inversa. Universitat Politècnica de València (2016). Proyecto clase inversa UPV. Recuperado de: http://claseinversa.blogs.upv.es/el-proyecto/proyecto-clase-inversa-upv-2/

Lage, M.J., Platt, G.J. Y Treglia, M. (2000). Inverting the classroom: A gateway to creating an inclusive learning environment. En The Journal of Economic Education, vol. 31, issue 1, 30-43.

Vidal Meló, A., Roig Sala, B., Estruch Fuster, V.D. y Boigues Planes, F.J. (2013). Una yincana para descubrir y generar curvas en un trabajo cooperativo. En Actas del XXI CUIEET, 950-961.

Vidal Meló, A., Boigues Planes, F.J., Estruch Fuster, V.D. (2017) Representando y disfrazando superficies. Aceptado para su publicación en INNODOCT 2017. 